Int. J. Dev. Biol. 57: 69-72 (2013)

doi: $10.1387 / \mathrm{ijdb} .120114 \mathrm{~mm}$

\title{
Innate sexuality determines the mechanisms of telomere maintenance
}

\author{
KENTA TASAKA ${ }^{1}$, NAOKI YOKOYAMA ${ }^{1}$, HANAE NODONO ${ }^{1}$, MOTONORI HOSHI ${ }^{2}$ and MIDORI MATSUMOTO ${ }^{*, 1}$ \\ ${ }^{1}$ Department of Biosciences and Informatics, Keio University, Hiyoshi, Kouhoku-ku, Yokohama, Japan and \\ ${ }^{2}$ The Open University of Japan, Wakaba, Mihama-ku, Chiba, Japan
}

\begin{abstract}
Recently, telomere length has been shown to be differentially regulated in asexually and sexually reproducing planarians. In addition, it was found that asexual worms maintain telomere length somatically during reproduction by fission or when regeneration is induced by amputation, whereas sexual worms only achieve telomere elongation through sexual reproduction. We have established an experimental bioassay system to induce switching from asexual to sexual reproduction in planarians, that is, sexualization. In this study, the relationship between the reproductive mode and telomere maintenance was investigated using innate asexually reproducing worms, innate sexually reproducing worms, and experimentally sexualized worms. Here, we show that innate asexual planarians maintain telomere length during cell division and that innate sexual planarians exhibit telomere shortening. However, experimental sexualized worms maintain telomere length during cell division. These results indicate that innate sexuality is linked to the mechanism of telomere maintenance.
\end{abstract}

KEY WORDS: telomere length, innate sexuality, planarian

Prokaryotes maintain an intact genome by asexual reproduction and therefore do not have a fixed lifespan. However, eukaryotic organisms that reproduce sexually produce offspring that have completely new genomes due to recombination of the parental genomes. In these organisms, cell lineages have a defined lifespan, as do the organisms themselves (Hug 2006; Williams 1957), which is linked to telomere shortening during each round of eukaryotic DNA replication. However, some eukaryotic animals can also reproduce asexually; therefore, asexual species, such as planarians, may not have a fixed lifespan.

The planarian is a useful model animal for investigating the relationship between the reproductive mode and predetermined lifespan. Many planarian species have strains with different modes of reproduction: exclusively asexual, exclusively sexual, or switching between them seasonally (Hyman, 1951). Although sexual planarians do not reproduce asexually (i.e., spontaneous fission), they can regenerate all tissues after amputation, including germ cells and somatic cells of the genital organs (Nodono et al., 2012). The mechanism controlling lifespan in these species has not been elucidated, but many extrinsic factors, including oxidative stress and regulation of energy production, and intrinsic factors, such as maintenance of telomere length, are expected to be involved. It was recently shown in the planarian Schmidtea mediterranea that somatic telomere maintenance differs between asexually and sexually reproducing animals. Telomere length is maintained somatically during asexual reproduction by fission or when regeneration is induced by amputation, whereas sexually reproducing animals can only achieve telomere elongation by sexual reproduction (Tan et al., 2012). This difference is reflected in the expression of alternatively spliced forms of the telomerase enzyme protein subunit. Therefore, asexually reproducing animals must have telomere-maintenance mechanisms that allow telomere maintenance to occur somatically.

We have established an experimental bioassay system to induce sexual switching in the planarian Dugesia ryukyuensis $\mathrm{OH}$ strain (Kobayashi et al., 1999), which has been maintained under laboratory conditions for more than 20 years through only asexual reproduction. Sexual reproduction can be induced by feeding with sexually mature planarian. We termed this experimentally sexualized worm "acquired sexual (AqS)" worm. When 2 AqS worms mate, they produce both exclusively asexually and sexually reproducing offspring. We termed innate asexual (AS) and innate sexual (InS) worms, respectively. Recently, we compared InS and AqS worm's stem cell capability by transplantation into X-ray irradiated AS re-

Abbreviations used in this paper: AqS, acquired sexual; AS, asexual; InS, innate sexual.

\footnotetext{
*Address correspondence to: Midori Matsumoto. Department of Biosciences and Informatics, Keio University, Hiyoshi, Kouhoku-ku, Yokohama, Japan. Tel: +81-45-566-1774. Fax: +81-45-566-1448. E-mail: mmatsumo@ bio.keio.ac.jp
} 
cipients, revealing that only InS worms can autonomously initiate a sexual state and AqS worms do not acquire the sexual state initiation capability like InS worms, although AqS worms can maintain the induced sexual state (Nodono and Matsumoto, 2012; Nodono et al., 2012). Therefore, these 2 sexually reproducing worms with different innate reproductive mode enable us to test the relationship between innate sexuality and telomere maintenance. In this study, we investigated whether sexually reproducing animals can only achieve telomere elongation through sexual reproduction.

\section{Results}

\section{Telomere FISH analysis in D. ryukyuensis}

We compared the karyotypes and morphological features of $D$. ryukyuensis planarian worm populations for different reproductive modes (AS vs. InS). The karyotypes of these worms included some chromosomal changes, including polyploidy and heteroploidy. The most typical triploid karyotypes of AS worms and InS worms are shown in Fig. 1A. These karyotypes are identical and do not show any translocations. FISH analysis of the (TAAGGG) ${ }_{4}$ sequence in triploid organisms of the $D$. ryukyuensis $\mathrm{OH}$ strain (Fig. 1B) showed that the chromosomal ends were fluorescently labeled.

\section{Telomere maintenance in asexual planarians}

To investigate whether AS worms maintain their telomere length, we performed Southern hybridization by using the telomere probe

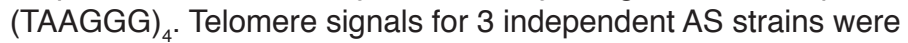
broad and smeared, with an average maximum length of $34.83 \mathrm{~kb}$ $(S D=0.76)$, an average minimum length of $13.17 \mathrm{~kb}(S D=0.76)$, and a mean length of $24.00 \mathrm{~kb},(\mathrm{SD}=0.75)$ (Fig. 2A). In addition, to determine whether telomere length was dependent on the period since hatching, telomere length was compared between newborn AS worms, AS worms maintained over 2 years with repeated amputation and regeneration, and $\mathrm{OH}$ worms (Fig. 2B). A mean telomere length of $24.00 \mathrm{~kb}$ was observed in all 3 populations.

\section{Telomere maintenance in innate sexual planarians}

Telomere length was compared between newborn InS worms, InS worms maintained for 2 years with repeated amputation and regeneration, and $\mathrm{OH}$ worms. Telomere length in InS worms de-

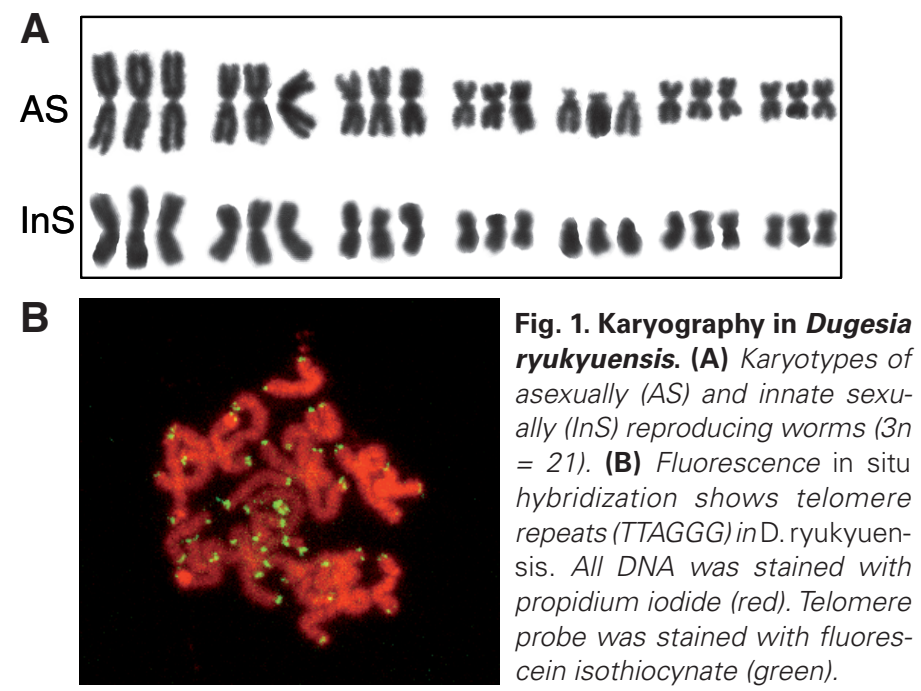

A

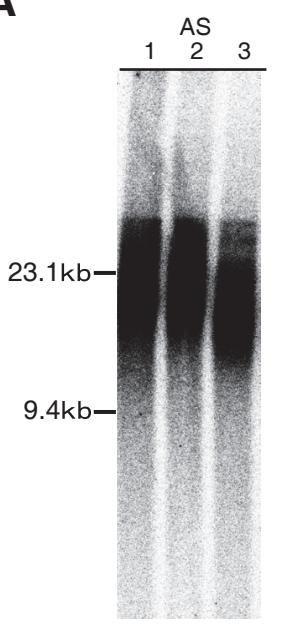

B

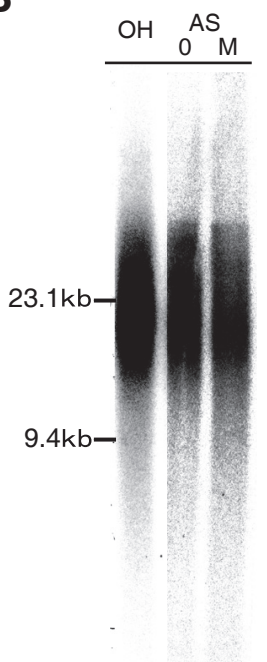

Fig. 2. Telomere length in asexual (AS) planarians. (A) Telomere length in 3 independent experimentally sexualized (AqS) strains. (B) Effect of aging on telomere length in AqS worms. 0 , newborn worms; $M$, worms maintained with repeated amputation and regeneration for 2 years; $\mathrm{OH}$, the D. ryukyuensis fissiparous $\mathrm{OH}$ strain.

pends on the period after hatching (mean length in newborn InS worms, $18.0 \mathrm{~kb}$; mean length in 2 year-old InS worms, $14.5 \mathrm{~kb}$; Fig. 3A). Statistical analysis showed that the range of telomere sizes in InS worms was wider than that in AS worms (AS worms, 1.036; InS worms, 8.060; $n=7$ for both) and the telomeres were significantly longer in AS worms than in InS worms (mean length in AS worms, $23.67 \mathrm{~kb}$; mean length in InS worms, $18.89 \mathrm{~kb} ; n=$ 7; two-tailed t-test, $P<0.003$ ).

Telomere length in InS worms shortened depending on the age, which led us to investigate the ability to maintain the telomere length during cell cycle. InS worms had been amputated and regenerated up to 12 times to activate the somatic cell cycle and the telomere length of InS worms regenerated 4, 8, and 12 times was examined (Fig. 3B). Worms that regenerated 4 times maintained a telomere length similar to that of asexually reproducing worms. However, the worms that regenerated 8 and 12 times had shorter telomeres. Repeated amputation and regeneration for 12 times shortened the InS worm's telomere by 200 bp (Fig. 3C). These data show that telomeres shorten in InS planarians by approximately 16.7 bp per regeneration.

\section{Telomere maintenance in acquired sexual planarians}

To investigate whether sexually reproducing animal can only achieve telomere elongation through sexual reproduction, telomere length was compared between newly sexualized AqS worms, AqS worms maintained for over 2 years with repeated amputation and regeneration, and $\mathrm{OH}$ worms. The telomere signals in 3 independent AqS strains were broad and smeared (Fig. 4A), with an average maximum length of $32.33 \mathrm{~kb}(\mathrm{SD}=2.31)$, an average minimum length of $15.33 \mathrm{~kb}(\mathrm{SD}=0.29)$, and a mean length of $23.83 \mathrm{~kb}$ $(S D=1.23)$. These telomeres are similar in length to telomeres in AS worms (mean length, $24.00 \mathrm{~kb}$ ). Statistical analysis showed that telomere length was not significantly different between AqS worms at several period after sexualization and in AS worms (mean length in AS worms, $24.00 \mathrm{~kb}$; mean length in AqS worms, 23.83 

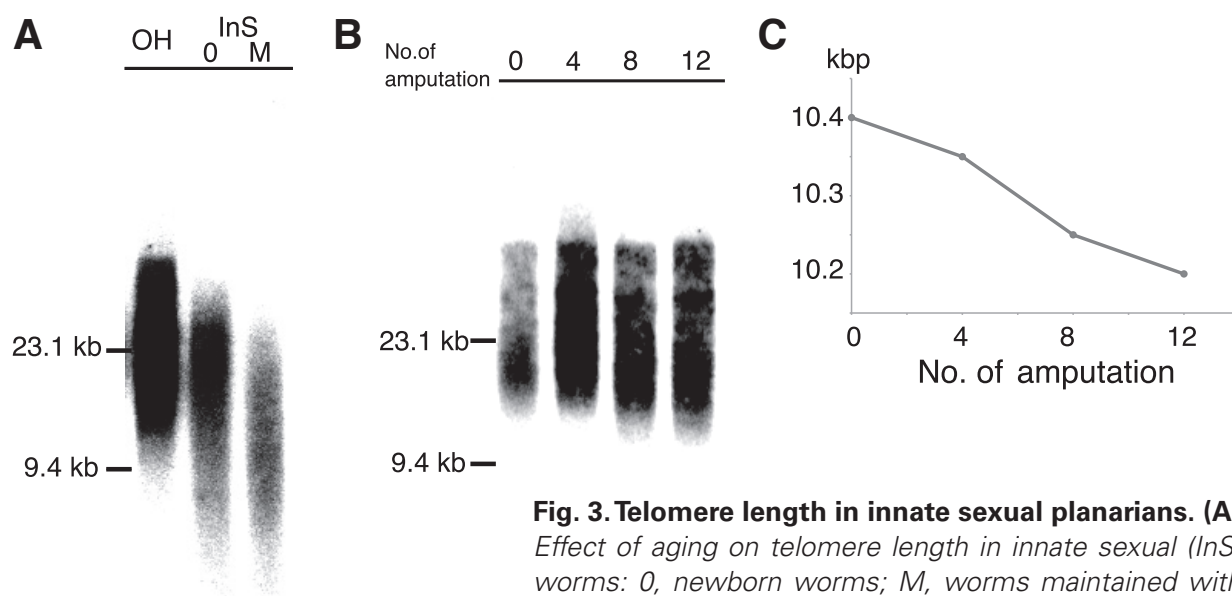

Fig. 3. Telomere length in innate sexual planarians. (A) Effect of aging on telomere length in innate sexual (InS) worms: 0, newborn worms; $M$, worms maintained with repeated amputation and regeneration for over 2 years.

(B) Effect of the number of amputation and regeneration cycles on telomere length in InS worms. (C) Graph showing data from Fig. 3B. The x-axis is the minimum length of telomere.

$\mathrm{kb} ; n=3$; two-tailed t-test, $P<0.85$ ). The telomere length did not differ between newly sexualized worms and worms with repeated amputation and regeneration (Fig. 4B).

\section{Discussion}

There are 3 reproductive strategies in planarians: exclusively asexual, exclusively sexual, and switching between the asexual and sexual modes (Kenk, 1937; Kobayashi and Hoshi, 2002). By comparing telomere length in the 3 reproductive modes (AS, InS, and AqS) in D. ryukyuensis, we found that AS worms can maintain telomere length during regeneration (Fig. 2) and that telomeres shorten during InS worm regeneration (Fig. 3). These data support previous reports that telomere maintenance differs in asexual worms and sexual worms in S. mediterranea (Tan et al., 2012). Our results show that the telomere length in AS worms that have reproduced by fission and regeneration for over 20 years is the same as that in AS newborn worms (Fig. 2B). However, it is not known whether switching the reproductive mode, resulting in the production of offspring by fertilization and embryogenesis, can help maintain telomere length. In mice and cattle, it has been demonstrated that a telomere-elongation program is initiated at the morula to blastocyst transition that establishes a specific telomere length set point during embryogenesis (Schaetzlein et al., 2004). AqS worms that have transformed from the asexual to the sexual reproductive mode by our method provide a useful experimental model to address this issue. In this study, we observed that AqS worms can maintain their telomere length almost as well as AS worms (Fig. 4). Therefore, the ability to produce germ cells and sexual organs is not linked to telomere maintenance.

However, it is not known whether the rate of change in telomere length is associated with lifespan. In birds and mammals, telomere length shortens more slowly in long-lived species than in shortlived ones (Haussmann, et al., 2003). For example, the maximum lifespan of zebra finches is 5 years, of tree swallows is 11 years, and of Adélie penguins is 20 years, and in these species, the rate of change in telomere length is $515 \mathrm{bp} / \mathrm{year}, 391 \mathrm{bp} / \mathrm{year}$, and 235 bp/year, respectively. In this study, InS worms showed a relationship between regeneration number and the telomere length, but $A S$ worms did not. Then, AS worms might not have a fixed life-span.
Neoblasts are proliferating cells in planarian bodies, comprising approximately $30 \%$ of their entire body (Baguñà, 1981; 2012). If it is assumed that the neoblasts in the half-body remaining after fission can proliferate and differentiate to resynthesize the entire body, then these neoblasts should each undergo cell division about 3 times. Therefore, neoblast telomeres should shorten by approximately 5 bp per cell division. In S. mediterranea, sexual and asexual strains can be distinguished by the presence of a chromosomal translocation only in asexually reproducing worms (Newmark and Sánchez Alvarado, 2002). Planarians containing this translocation reproduce by transverse fission and do not show differentiation for a germ line or somatic copulatory apparatus; planarians lacking this translocation are hermaphrodites that do not reproduce asexually. Asexually reproducing worms can produce offspring only by fission and regeneration, and only sexual reproducing worms can produce differentiated germ cells and perform fertilization. $S$. mediterranea worms with different reproductive modes can be distinguished by a chromosome translocation, i.e., both asexual and sexual strains are different. However, in D. ryukyuensis, the chromosome patterns are not different in both strains (Fig.1A). And worms can naturally switch the mode of reproduction to either sexual or asexual. AqS worms, which are transformed from asexual to sexual worms, produce offspring that reproduce either asexually or sexually. In addition, AqS worms can reproduce by genetic cross-mixing (Kobayashi et al., 2008).

Recently, we reported that neoblasts from InS worms but not AqS worms have the autonomous capability to form individuals that can reproduce sexually (Nodono and Matsumoto 2012; Nodono et
A

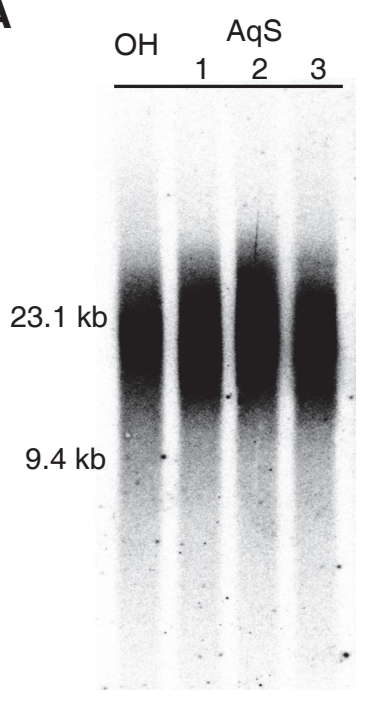

B

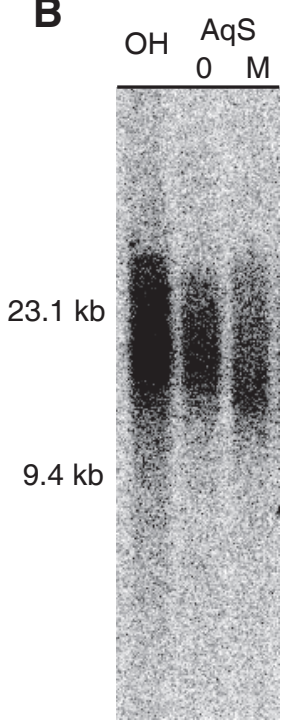

Fig. 4. Telomere length in acquired sexual planarians. (A) Telomerelength in 3 independent AqS strains. O, newborn worms; $M$, worms maintained with repeated amputation and regeneration for 2 years. (B) Effect of aging on telomere length in experimentally sexualized (AqS) worms. 


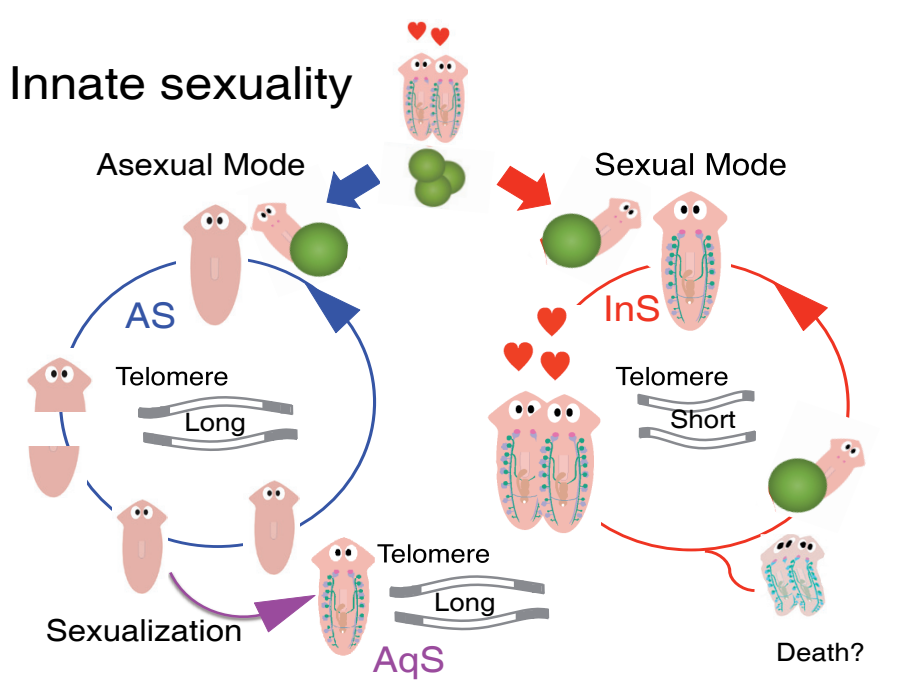

Fig. 5. Schematic of the relation of telomere length and innate sexuality.

al., 2012). In the 2 types of planarians that produce sexual organs, cellular abilities differ according to the sexual mode they have at birth (i.e., their innate sexuality). In this study, we showed that AqS worms can maintain telomere length and that InS worms cannot maintain telomere length. Therefore, the ability to maintain telomere length is determined by the mode of reproduction at birth (Fig.5).

In $S$. mediterranea, the capacity to maintain telomere length is determined by the expression level and alternative splicing of the telomerase enzyme protein subunit (Tan et al., 2012). D. ryukyuensis is a useful model species for studying switching of reproductive modes. Studying the mechanism of telomerase gene expression in D. ryukyuensis (AS, AqS, and InS) could improve our understanding of the relationship between the reproductive mode and lifespan.

\section{Materials and Methods}

\section{Planarian strains and culture}

The planarian $D$. ryukyuensis $\mathrm{OH}$ strain was provided by Dr. S. Ishida of Hirosaki University. AS worms and InS worms were cultured as previously described (Kobayashi et al., 2009). All the AqS worms were obtained by feeding $\mathrm{OH}$ worms with Bdellocephala brunnea worms (Kobayashi et al., 1999).

\section{Chromosome preparation and fluorescence in situ hybridization}

To prepare metaphase spreads of mitotic chromosomes for imaging, a previously published hybridization protocol was followed (Kobayashi et al., 2008). The preparations were dried overnight and stored at $-80^{\circ} \mathrm{C}$. The samples were stained with Giemsa stain or used for fluorescence in situ hybridization (FISH). Studies on the Planariidae Polycelis tenuis and the Dendrocoelidae Dendrocoelum lacteum have indicated that the planarian telomere sequence is a repeated TTAGGG sequence (Joffe et al., 1996, 1998). A previously published hybridization protocol was followed (Suzuki, et al., 1999), using the labeled telomere probe (TTAGGG) ${ }_{4}$.

\section{DNA preparation and Southern blot analysis}

Planarian genomic DNA was prepared using the Wizard SV Genomic DNA preparation system (Promega, USA) according to the manufacturer's instructions. For Southern blot analysis, genomic DNA, completely digested by Hinfl, was separated on a $0.5 \%$ agarose gel and hybridized with the telomere probe (TTAGGG) by using previously described protocols (Tan et al., 2012).

\section{Acknowledgments}

We would like to thank F. Ishikawa, A. Matsuura and K. Kobayashi for their valuable comments on these experiments, $S$. Ishida for the gift of $D$. ryukyuensis $\mathrm{OH}$ strain, and S. Arioka for karyotypic analysis. This work was partly supported by a Grant-in-Aid for Scientific Research on Priority Areas, "Mechanisms of Sex Differentiation" (No. 16086209), to M. M. from the Ministry of Education, Culture, Sports, Science and Technology, Japan, and a Grant-in-Aid from the Takeda Science Foundation.

\section{References}

BAGUÑÀ J (1981). Planarian neoblast. Nature 290: 14-15.

BAGUÑÀ J (2012). The planarian neoblast: the rambling history of its origin and some current black boxes. Int J Dev Biol 56: 19-37.

HAUSSMANN M, WINKLER D, O'REILLY K, HUNTINGTON C, NISBET I, VLECK C (2003). Telomeres shorten more slowly in long-lived birds and mammals than in short-lived ones. Proc Biol Sci 270: 1387-1392.

HUG N, LINGNERJ (2006). Telomere length homeostasis. Chromosoma 115:413-425.

HYMAN L (1951). Platyhelminthes and Rhynchocoela. In The Invertebrates II. McGraw-Hill, New York, pp. 52-219.

JOFFE BI, SOLOVEI IV, MACGREGOR HC (1996). Ends of chromosomes in Polycelis tenuis (Platyhelminthes) have telomere repeat TTAGGG. Chromosome Res 4: 323-324

JOFFE BI, SOLOVEI IV, MACGREGOR HC (1998). Ordered arrangement and rearrangement of chromosomes during spermatogenesis in two species of planarians (Plathelminthes). Chromosoma 107: 173-183.

KENK R (1937). Sexual and asexual reproduction in Euplanaria tigrina (Girard). Biol Bull 73: 280-294

KOBAYASHIK, ARIOKAS, HOSHI M, MATSUMOTO M (2009). Production of asexual and sexual offspring in the triploid sexual planarian Dugesia ryukyuensis. Integr Zool 4: 265-271.

KOBAYASHI K, ISHIZU H, ARIOKA S, CABRERA JP, HOSHI M, MATSUMOTO M (2008) Production of diploid and triploid offspring by inbreeding of the triploid planarian Dugesia ryukyuensis. Chromosoma 117: 289-296.

KOBAYASHI K, KOYANAGI R, MATSUMOTO M, CABRERA JP, HOSHI M (1999) Switching from asexual to sexual reproduction in the planarian Dugesia ryukyuensis: Bioassay system and basic description of sexualizing process. Zoolog Sci 16: $291-298$

NEWMARK PA, SÁNCHEZ ALVARADO A (2002). Not your father's planarian: a classic model enters the era of functional genomics. Nat Rev Genet 3: 210-219.

NODONO H, ISHINO Y, HOSHI M, MATSUMOTO M (2012). Stem cells from innate sexual but not acquired sexual planarians have the capability to form a sexual individual Mol Reprod Dev 79: 757-766.

NODONO H, MATSUMOTO M (2012). Reproductive mode and ovarian morphology regulation in chimeric planarians composed of asexual and sexual neoblasts. Mol Reprod Dev 79: 451-460.

SCHAETZLEIN S, LUCAS-HAHN A, LEMME E, KUES W, DORSCH M, MANNS M, NIEMANN H, RUDOLPH K (2004). Telomere length is reset during early mammalian embryogenesis. Proc Natl Acad Sci USA 101: 8034-8038.

SUZUKI T, KUROSAKI T, SHIMADA K, KANSAKU N, KUHNLEIN U, ZADWORNY D, AGATA K, HASHIMOTO A, KOIDE M, KOIKE M, TAKATA M, KUROIWA A, MINAI S, NAMIKAWA T, MATSUDA Y (1999). Cytogenetic mapping of 31 functional genes on chicken chromosomes by direct R-banding FISH. Cytogene Cell Genet 87: 32-40.

TANTC, RAHMANR, JABER-HIJAZI F, FELIXDA, CHENC, LOUISEJ, ABOOBAKER A (2012). Telomere maintenance and telomerase activity are differentially regulated in asexual and sexual worms. Proc Natl Acad Sci U S A 109: 4209-4214.

WILLIAMS GC (1957). Pleiotropy, Natural selection, and the evolution of senescence. Evolution 11: 398-411. 


\section{Further Related Reading, published previously in the Int. J. Dev. Biol.}

Comprehensive gene expression analyses in pluripotent stem cells of a planarian, Dugesia japonica

Norito Shibata, Tetsutaro Hayashi, Ryutaro Fukumura, Junsuke Fujii, Tomomi Kudome-Takamatsu, Osamu Nishimura, Syozo Sano, Fuyan Son, Nobuko Suzuki, Ryoko Araki, Masumi Abe and Kiyokazu Agata

Int. J. Dev. Biol. (2012) 56: 93-102

The planarian P2X homolog in the regulation of asexual reproduction

Toshihide Sakurai, Hayoung Lee, Makoto Kashima, Yumi Saito, Tetsutaro Hayashi, Tomomi

Kudome-Takamatsu, Osamu Nishimura, Kiyokazu Agata and Norito Shibata

Int. J. Dev. Biol. (2012) 56: 173-182

The Dr-nanos gene is essential for germ cell specification in the planarian Dugesia ryukyuensis

Haruka Nakagawa, Hirotsugu Ishizu, Ayako Chinone,Kazuya Kobayashi and Midori Matsumoto

Int. J. Dev. Biol. (2012) 56: 165-171

Diverse miRNA spatial expression patterns suggest important roles in homeostasis and regeneration in planarians

Cristina González-Estévez, Varvara Arseni, Roshana S. Thambyrajah, Daniel A. Felix and A. Aziz Aboobaker

Int. J. Dev. Biol. (2009) 53: 493-505

Characterization of novel genes expressed specifically in the sexual organs of the planarian Dugesia ryukyuensis

Sumitaka Hase, Emiko Kashiwagi, Kazuya Kobayashi, Motonori Hoshi and Midori Matsumoto Int. J. Dev. Biol. (2007) 51: 345-349

5 yr ISI Impact Factor $(2011)=2.959$

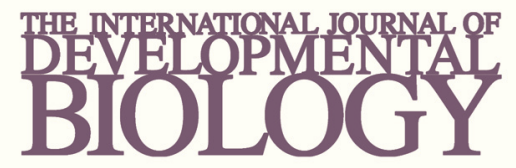

Volume 54 Nos. $6 / 7$
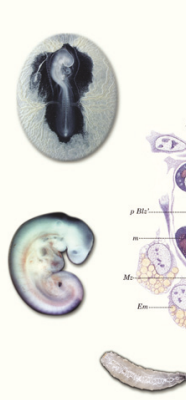

Developmental Hematopoiesis
Special Issue

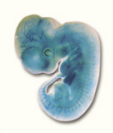

(2) (1)

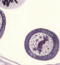

(1)
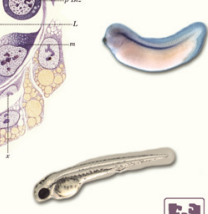

5

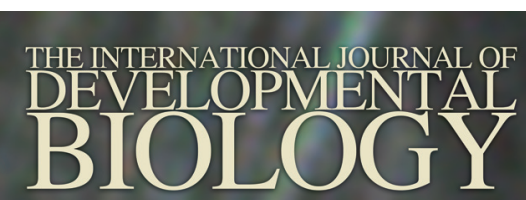

Special Issue

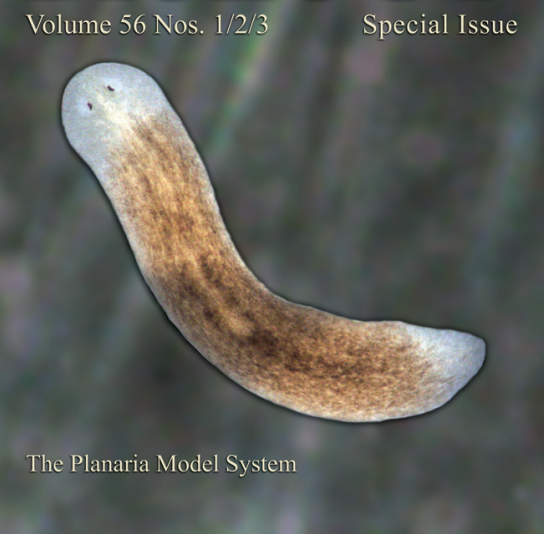

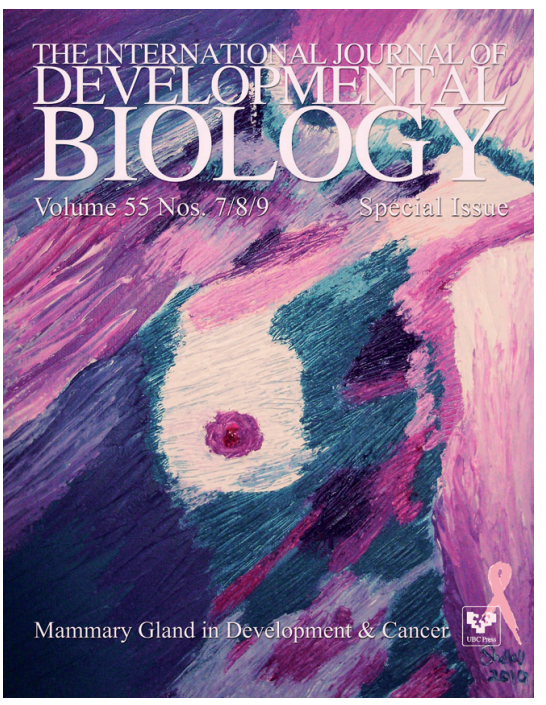

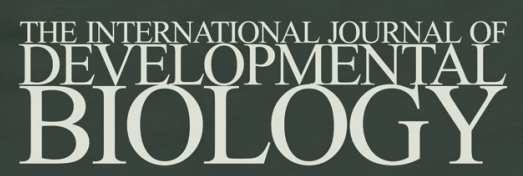

Volume 55 Nos. $4 / 5$

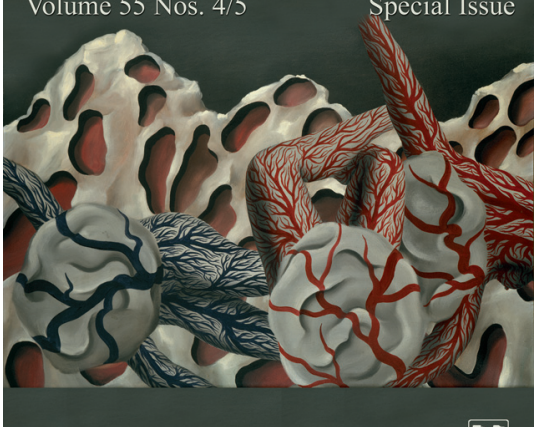

Angiogenesis in Development \& Cancer 